\title{
Three acylated flavone glycosides from Sideritis ozturkii Aytac \& Aksoy
}

\author{
F. Pınar Şahin ${ }^{\mathrm{a}, *}$, Nurten Ezer ${ }^{\mathrm{a}}$, İhsan Çalış ${ }^{\mathrm{b}}$ \\ ${ }^{\text {a }}$ Faculty of Pharmacy, Department of Pharmaceutical Botany, Hacettepe University, 06100 Ankara, Turkey \\ ${ }^{\mathrm{b}}$ Faculty of Pharmacy, Department of Pharmacognosy, Hacettepe University, 06100 Ankara, Turkey
}

Received 12 December 2003; accepted 3 March 2004

Available online 8 May 2004

\begin{abstract}

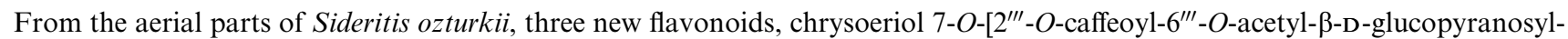
$(1 \rightarrow 2)$ - $\beta$-D-glucopyranoside], chrysoeriol 7-O-[2"'-O-caffeoyl- $\beta$-D-glucopyranosyl- $(1 \rightarrow 2)-\beta$-D-glucopyranoside] and chrysoeriol

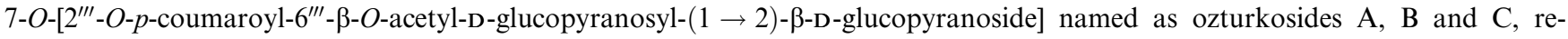
spectively, were isolated, along with three known phenylethanoid glycosides, verbascoside, leucoseptoside A, martynoside and five known diterpenoids, 7-epicandicandiol, linearol, sidol, sideroxol, epoxyisolinearol. The structures were elucidated mainly by spectroscopic methods.
\end{abstract}

(c) 2004 Elsevier Ltd. All rights reserved.

Keywords: Sideritis ozturkii; Lamiaceae; Flavonoids; Acylated flavone glycosides; Phenylethanoids; Diterpenoids

\section{Introduction}

The genus Sideritis L. (Lamiaceae) is represented by more than 150 species which are distributed especially in Mediterranean region (Obon de Castro and RiveraNunez, 1994). In Turkey 46 species are growing (Aytac and Aksoy, 2000) and some of which have been used in traditional medicine and as herbal tea (Baytop, 1999). The genus is known to be rich in essential oils (Ezer et al., 1996; Baser, 2002), diterpenes (Topcu et al., 2002), flavonoids and phenylethanoid glycosides (Ezer et al., 1992; Ezer and Akcos, 1995; Akcos et al., 1999). As a part of our continuing phytochemical studies on Sideritis species, here in we report the isolation and structure elucidation of three new flavonoids, named ozturkosides A, B, C (1-3), from the acetone extract of the aerial parts of S. ozturkii Aytac \& Aksoy together with known phenylethanoid glycosides (verbascoside, leucoseptoside A, martynoside) and diterpenoids (7-epicandicandiol,

\footnotetext{
${ }^{*}$ Corresponding author. Tel.: +90-312-305-10-89; fax: +90-312-31147-77.

E-mail address: psahin@hacettepe.edu.tr (F. Pınar Şahin).
}

linearol, sidol, sideroxol, epoxyisolinearol). S. ozturkii is an endemic species and a new record for the flora of Turkey.

\section{Results and discussion}

VLC, repeated column chromatography (polyamide and silica gel) and MPLC of acetone extract of the aerial parts of $S$. ozturkii yielded compounds 1-11 (Fig. 1). Compound 1 was isolated as an amorphous yellow powder. The ${ }^{1} \mathrm{H}$ and ${ }^{13} \mathrm{C}$ NMR spectra of 1 demonstrated the presence of aromatic systems, sugar moieties and olefinic protons. The UV spectrum exhibited maxima at $250(\mathrm{sh}), 270,337 \mathrm{~nm}$ and spectroscopic data with diagnostic reagents were indicative of a flavone skeleton (Mabry et al., 1970). The IR spectrum indicated absorption bands for hydroxyl $\left(3353 \mathrm{~cm}^{-1}\right)$, ester carbonyl $\left(1715 \mathrm{~cm}^{-1}\right), \gamma$-pyrone carbonyl $\left(1657 \mathrm{~cm}^{-1}\right)$ and aromatic rings $\left(1602,1516 \mathrm{~cm}^{-1}\right)$. The negative ion mode ESI mass spectrum showed a signal at $m / z 827[\mathrm{M}-\mathrm{H}]^{-}$, compatible with the molecular formula $\mathrm{C}_{39} \mathrm{H}_{40} \mathrm{O}_{20}$. In the ${ }^{1} \mathrm{H}$ NMR spectrum of $\mathbf{1}$ (Table 1) the presence of 

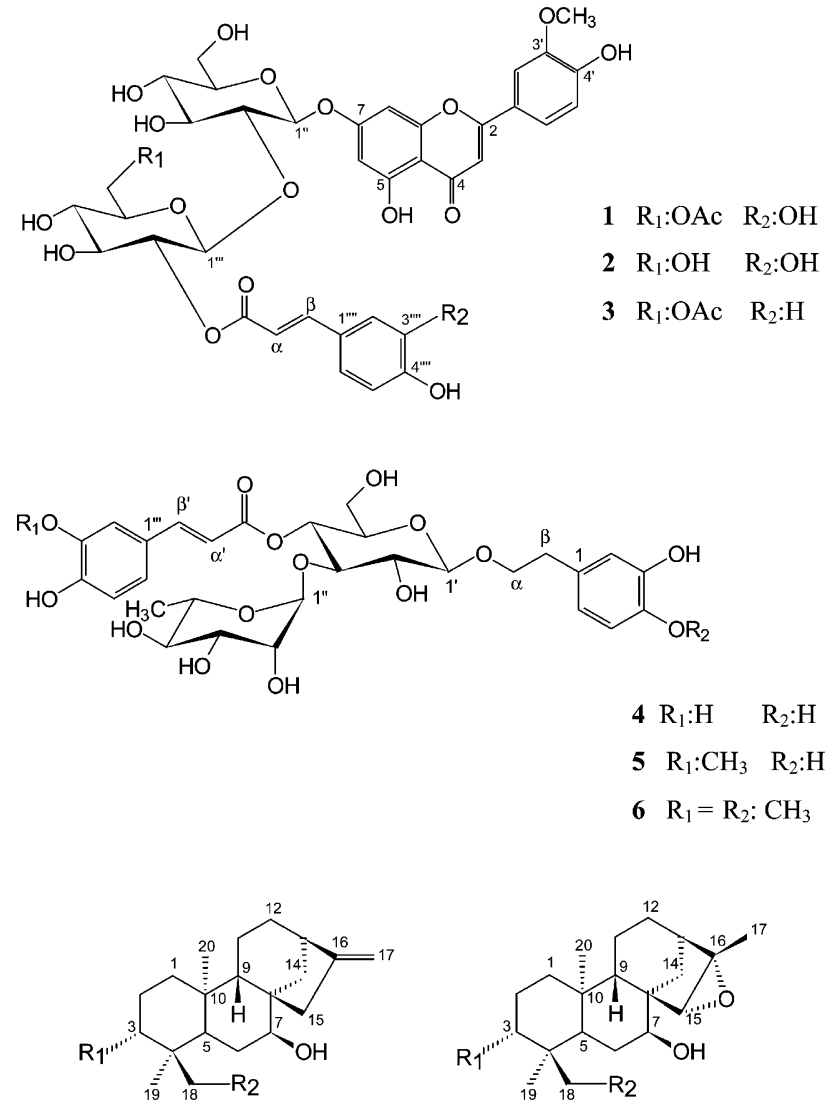

$$
\begin{array}{llllll}
7 & \mathrm{R}_{1}: \mathrm{H} & \mathrm{R}_{2}: \mathrm{OH} & \mathbf{1 0} & \mathrm{R}_{1}: \mathrm{H} & \mathrm{R}_{2}: \mathrm{OH} \\
\mathbf{8} & \mathrm{R}_{1}: \mathrm{OH} & \mathrm{R}_{2}: \mathrm{OAc} & \mathbf{1 1} & \mathrm{R}_{1}: \mathrm{OH} & \mathrm{R}_{2}: \mathrm{OAc}
\end{array}
$$

Fig. 1. Compounds isolated from Sideritis ozturkii.

disubtituted ring $\mathrm{B}$ was confirmed by an $\mathrm{ABX}$ pattern signals at $\delta_{\mathrm{H}} 7.58\left(\mathrm{~d}, J=2.2 \mathrm{~Hz}, \mathrm{H}-2^{\prime}\right), 7.56(\mathrm{dd}$, $\left.J=2.2,8.2 \mathrm{~Hz}, \mathrm{H}-6^{\prime}\right)$ and 6.94 (H-5). Furthermore, in the aromatic region, two meta-coupled doublets at $\delta_{\mathrm{H}}$ $6.42(\mathrm{~d}, J=2.0 \mathrm{~Hz}, \mathrm{H}-6)$ and $6.76(\mathrm{~d}, J=2.0 \mathrm{~Hz}, \mathrm{H}-8)$ were characteristic of the 5,7-disubstituted A ring of flavonoid. Singlet signal at $\delta_{\mathrm{H}} 6.93$ was attributed to $\mathrm{H}$ 3. In the ${ }^{1} \mathrm{H}$ NMR spectrum of $\mathbf{1}$, signals at $\delta_{\mathrm{H}} 3.90(\mathrm{~s}$, $3 \mathrm{H})$ and $2.00(\mathrm{~s}, 3 \mathrm{H})$ also revealed the presence of a methoxyl group and acetoxymethyl function, respectively. Unambiguous assignment for the ${ }^{1} \mathrm{H}$ and ${ }^{13} \mathrm{C}$ NMR signals in 1 were made by combination of the DQF-COSY, HSQC, HMBC and ROESY experiments. Therefore, according to established NMR data of $\mathbf{1}$, the structure of the aglycone moiety was shown to be chrysoeriol (Calis et al., 2002). Two anomeric proton resonances of the disaccharide at $\delta_{\mathrm{H}} 5.20(\mathrm{~d}, J=7.8)$ and $4.91(\mathrm{~d}, J=8.1)$ were used in conjunction with DQF-COSY and HSQC data to assign the ${ }^{1} \mathrm{H}$ and ${ }^{13} \mathrm{C}$ NMR resonances of sugars and identified both of them as $\beta$-glucopyranose (Klimek, 1988). The downfield of $\mathrm{C}-2^{\prime \prime}$ of terminal glucose to $\delta_{\mathrm{C}} 80.1$ characterized the interglycosidic linkage between these sugars $(1 \rightarrow 2)$.
The ${ }^{13} \mathrm{C}$ NMR and DEPT-135 spectra of 1 showed, 39 carbon resonances $\left(14 \mathrm{C}, 21 \mathrm{CH}, 2 \mathrm{CH}_{2}\right.$ and $\left.2 \mathrm{CH}_{3}\right), 16$ of which could be assigned to chrysoeriol, 12 for two hexosyl units and 2 for an acetyl function. The remaining 9 resonances were consistent with the presence of caffeic acid (Klimek, 1988). The ${ }^{1} \mathrm{H}$ NMR spectrum of 1 showed three aromatic protons as an $\mathrm{ABX}$ system $\left[\delta_{\mathrm{H}} 7.02\left(\mathrm{~d}, J=2.0 \mathrm{~Hz}, \mathrm{H}-2^{\prime \prime \prime \prime}\right), 6.74(\mathrm{~d}, J=8.1 \mathrm{~Hz}, \mathrm{H}-\right.$ $\left.\left.5^{\prime \prime \prime \prime}\right), 6.95\left(\mathrm{dd}, J=2.0,8.1 \mathrm{~Hz}, \mathrm{H}-6^{\prime \prime \prime \prime}\right)\right]$ and two olefinic protons at $\delta_{\mathrm{H}} 6.24 \mathrm{~d}$ and $7.44 \mathrm{~d}\left(J_{\mathrm{AX}}=15.9 \mathrm{~Hz}\right.$, indicating trans-geometry) which appeared as an AX system were characteristic of trans-caffeoyl. The significant deshielding of $\mathrm{H}-2^{\prime \prime \prime}$ of terminal glucose $\left(\delta_{\mathrm{H}} 4.61\right)$ and HMBC cross-peak between this proton and the carbonyl carbon of caffeic acid at $\delta_{\mathrm{C}} 165.9$ confirmed that the caffeoyl residue was connected at $\mathrm{H}-2^{\prime \prime \prime}$ via ester linkage. Additionally, the long-range correlations between the anomeric proton of the terminal glucose $\left(\delta_{\mathrm{H}}\right.$ $\left.4.91, \mathrm{H}-1^{\prime \prime \prime}\right)$ and $\mathrm{C}-2^{\prime \prime}$ of inner glucose $\left(\delta_{\mathrm{C}} 80.1\right)$, and the anomeric proton of the inner glucose $\left(\delta_{\mathrm{H}} 5.20, \mathrm{H}-1^{\prime \prime}\right)$ and C-7 of chrysoeriol $\left(\delta_{\mathrm{C}} 162.7\right)$ demonstrated the $(1 \rightarrow 2)$ interglycosidic linkage between two glucosyl units and the site of glycosidation at C-7 of chrysoeriol moiety. HMBC spectrum also indicated the long-range couplings between carbonyl carbon of acetoxyl function $\left(\delta_{\mathrm{C}} 170.4\right)$ and H-6 $6^{\prime \prime \prime}$ representing the hydroxyl group at C- $6^{\prime \prime \prime}$ of terminal glucose was involved in the ester linkage. On the other hand, the methoxyl singlet at $\left(\delta_{\mathrm{H}}\right.$ 3.90) was correlated with $\mathrm{C}-3^{\prime}$ carbon resonance $\left(\delta_{\mathrm{C}}\right.$ 148.1) showing the attachment of the methoxyl group at C- $3^{\prime}$ of ring $\mathrm{B}$. In the ROESY spectrum, ROE correlations between the anomeric proton of the inner glucose and $\mathrm{H}-6$ and $\mathrm{H}-8$ protons of the aglycone, the anomeric proton of the terminal glucose and $\mathrm{H}-2^{\prime \prime}$ confirmed the suggested structure. Based on these data, the structure of compound 1 was established as chrysoeriol 7-O$\left[2^{\prime \prime \prime}\right.$ - $O$-caffeoyl- $6^{\prime \prime \prime}$ - $O$-acetyl- $\beta$-D-glucopyranosyl- $(1 \rightarrow 2)$ $\beta$-D-glucopyranoside], for which the trivial name ozturkoside A is proposed.

Compounds $\mathbf{2}$ and $\mathbf{3}$ were obtained as amorphous yellow powders. The molecular formulas of them were determined to be $\mathrm{C}_{37} \mathrm{H}_{38} \mathrm{O}_{19}$ and $\mathrm{C}_{39} \mathrm{H}_{40} \mathrm{O}_{19}$, respectively, by negative ion mode ESI mass spectra $(\mathrm{m} / \mathrm{z}$ $785[\mathrm{M}-\mathrm{H}]^{-}$and $811[\mathrm{M}-\mathrm{H}]^{-}$, respectively), ${ }^{13} \mathrm{C}$ NMR and DEPT. The UV absorptions recorded with shifting reagents $\left(\lambda_{\max } 250\right.$ (sh), 270, 336 and $250(\mathrm{sh})$, $271,330 \mathrm{~nm}$, respectively) and the IR spectrum were very similar to those of compound $\mathbf{1}$. When comparing their NMR data (Table 1), a striking resemblance was noticed between $\mathbf{1}$ and $\mathbf{2}$. The only difference was the absence of the acetyl signals in the spectra of $\mathbf{2}$. This assumption was proved by the chemical shift value of $\mathrm{C}-6^{\prime \prime \prime}\left(\delta_{\mathrm{C}} 60.5\right)$ resonance of the terminal glucose moiety, exhibiting no unusual shift due to an acylation. The NMR data of 3 (Table 1) showed that it possessed identical aglycone and sugar structures to 
Table 1

${ }^{13} \mathrm{C}$ and ${ }^{1} \mathrm{H}$ NMR spectroscopic data ${ }^{\mathrm{a}}$ for $\mathbf{1}-\mathbf{3}\left(\mathrm{DMSO}_{\mathrm{d}} \mathrm{d}_{6}\right)$

\begin{tabular}{|c|c|c|c|c|c|c|}
\hline \multirow{2}{*}{$\begin{array}{l}\mathrm{C} / \mathrm{H} \\
\text { Atom }\end{array}$} & \multicolumn{3}{|c|}{$\delta_{\mathrm{C}} \mathrm{ppm}$} & \multicolumn{3}{|l|}{$\delta_{\mathrm{H}} \operatorname{ppm}(J, \mathrm{~Hz})$} \\
\hline & 1 & 2 & 3 & 1 & 2 & 3 \\
\hline \multicolumn{7}{|c|}{ Aglycone } \\
\hline 2 & 164.2 & 164.1 & 164.2 & & & \\
\hline 3 & 103.5 & 103.4 & 103.4 & $6.93 \mathrm{~s}$ & $6.93 \mathrm{~s}$ & $6.94 \mathrm{~s}$ \\
\hline 4 & 182.1 & 182.0 & 182.0 & & & \\
\hline 5 & 161.2 & 161.1 & 161.1 & & & \\
\hline 6 & 99.3 & 99.6 & 99.3 & $6.42 \mathrm{~d}(2.0)$ & $6.46 \mathrm{~d}(2.0)$ & $6.42 \mathrm{~d}(2.0)$ \\
\hline 7 & 162.7 & 162.7 & 162.6 & & & \\
\hline 8 & 94.8 & 95.1 & 94.8 & $6.76 \mathrm{~d}(2.0)$ & $6.85 \mathrm{~d}(2.0)$ & $6.74 \mathrm{~d}(2.0)$ \\
\hline 9 & 156.9 & 156.9 & 156.9 & & & \\
\hline 10 & 105.3 & 105.3 & 105.3 & & & \\
\hline $1^{\prime}$ & 121.2 & 121.3 & 121.1 & & & \\
\hline $2^{\prime}$ & 110.3 & 110.3 & 110.2 & $7.58 \mathrm{~d}(2.2)$ & $7.56 \mathrm{~d}(2.0)$ & $7.56^{\mathrm{b}}$ \\
\hline $3^{\prime}$ & 148.1 & 148.1 & 148.1 & & & \\
\hline $4^{\prime}$ & 151.0 & 151.1 & 151.2 & & & \\
\hline $5^{\prime}$ & 115.8 & 115.8 & 115.7 & $6.94^{\mathrm{b}}$ & $6.94 \mathrm{~d}(8.0)$ & $6.94 \mathrm{~d}(7.7)$ \\
\hline $6^{\prime}$ & 120.5 & 120.5 & 120.5 & $7.56 \mathrm{dd}(2.2,8.2)$ & $7.57 \mathrm{dd}(2.0,8.0)$ & $7.57^{\mathrm{b}}$ \\
\hline $\mathrm{OCH}_{3}$ & 56.0 & 56.3 & 56.9 & $3.90 \mathrm{~s}$ & $3.89 \mathrm{~s}$ & $3.89 \mathrm{~s}$ \\
\hline \multicolumn{7}{|l|}{ Glucose } \\
\hline $1^{\prime \prime}$ & 97.5 & 97.7 & 97.3 & $5.20 \mathrm{~d}(7.8)$ & $5.20 \mathrm{~d}(8.1)$ & $5.20 \mathrm{~d}(7.8)$ \\
\hline $2^{\prime \prime}$ & 80.1 & 79.6 & 80.0 & 3.52 dd $(7.8,8.9)$ & 3.56 dd $(8.1,9.5)$ & $3.50 \mathrm{dd}(7.8,8.8)$ \\
\hline $3^{\prime \prime}$ & 76.1 & 75.9 & 75.9 & $3.38^{\mathrm{b}}$ & $3.40^{\mathrm{b}}$ & $3.37^{\mathrm{b}}$ \\
\hline $4^{\prime \prime}$ & 69.9 & 69.9 & 69.9 & $3.26^{\mathrm{b}}$ & $3.22^{\mathrm{b}}$ & $3.25^{\mathrm{b}}$ \\
\hline $5^{\prime \prime}$ & 76.7 & 76.7 & 76.7 & $3.46^{\mathrm{b}}$ & $3.44^{\mathrm{b}}$ & $3.44^{\mathrm{b}}$ \\
\hline \multirow[t]{2}{*}{$6^{\prime \prime}$} & 60.4 & 60.6 & 60.4 & $3.45 \mathrm{dd}(4.6,10.5)$ & $3.49 \mathrm{dd}(4.8,11.5)$ & $3.44^{\mathrm{b}}$ \\
\hline & & & & $3.68^{\mathrm{b}}$ & $3.58^{\mathrm{b}}$ & 3.66 br d (12.0) \\
\hline \multicolumn{7}{|l|}{ Glucose } \\
\hline $1^{\prime \prime \prime}$ & 100.7 & 100.1 & 100.4 & $4.91 \mathrm{~d}(8.1)$ & $4.92 \mathrm{~d}(8.1)$ & $4.91 \mathrm{~d}(8.1)$ \\
\hline $2^{\prime \prime \prime}$ & 73.7 & 73.9 & 73.6 & $4.61 \mathrm{dd}(8.1,9.4)$ & 4.59 dd $(8.1,9.5)$ & $4.61 \mathrm{dd}(8.1,9.3)$ \\
\hline $3^{\prime \prime \prime}$ & 74.1 & 74.5 & 74.1 & $3.43^{\mathrm{b}}$ & $3.41^{\mathrm{b}}$ & $3.43^{\mathrm{b}}$ \\
\hline $4^{\prime \prime \prime}$ & 69.8 & 69.7 & 69.7 & $3.20^{\mathrm{b}}$ & $3.17^{\mathrm{b}}$ & $3.18^{\mathrm{b}}$ \\
\hline $5^{\prime \prime \prime}$ & 73.5 & 76.8 & 73.5 & $3.44^{\mathrm{b}}$ & $3.19^{\mathrm{b}}$ & $3.45^{\mathrm{b}}$ \\
\hline \multirow[t]{2}{*}{$6^{\prime \prime \prime}$} & 63.1 & 60.5 & 63.1 & $4.13^{\mathrm{b}}$ & $3.65 \mathrm{dd}(2.0,12.2)$ & $4.13 \mathrm{dd}(2.0,12.2)$ \\
\hline & & & & $4.19 \mathrm{dd}(1.9,12.2)$ & $3.43^{\mathrm{b}}$ & 4.20 br d (12.2) \\
\hline \multicolumn{7}{|c|}{ Caffeic (Coumaric) acid } \\
\hline $1^{\prime \prime \prime \prime \prime}$ & 125.7 & 125.6 & 125.2 & & & \\
\hline $2^{\prime \prime \prime \prime}$ & 114.8 & 114.7 & 130.1 & $7.02 \mathrm{~d}(2.0)$ & $6.99 \mathrm{~d}(1.8)$ & $7.49 \mathrm{~d}(8.5)$ \\
\hline $3^{\prime \prime \prime \prime}$ & 148.2 & 148.3 & 115.7 & & & $6.79 \mathrm{~d}(8.5)$ \\
\hline $4^{\prime \prime \prime \prime \prime}$ & 145.6 & 145.6 & 159.7 & & & \\
\hline $5^{\prime \prime \prime \prime}$ & 115.7 & 115.7 & 115.7 & $6.74 \mathrm{~d}(8.1)$ & $6.71 \mathrm{~d}(8.1)$ & $6.79 \mathrm{~d}(8.5)$ \\
\hline $6^{\prime \prime \prime \prime}$ & 121.4 & 121.1 & 130.1 & $6.95 \mathrm{dd}(2.0,8.1)$ & $6.92 \mathrm{dd}(1.8,8.1)$ & $7.49 \mathrm{~d}(8.5)$ \\
\hline$\alpha$ & 114.7 & 114.6 & 114.8 & $6.24 \mathrm{~d}(15.9)$ & $6.20 \mathrm{~d}(15.9)$ & $6.32 \mathrm{~d}(15.9)$ \\
\hline$\beta$ & 144.6 & 144.5 & 144.2 & $7.44 \mathrm{~d}(15.9)$ & $7.40 \mathrm{~d}(15.9)$ & $7.50 \mathrm{~d}(15.9)$ \\
\hline $\mathrm{C}=\mathrm{O}$ & 165.9 & 165.8 & 165.9 & & & \\
\hline $\mathrm{COCH}_{3}$ & 20.7 & & 20.6 & $2.00 \mathrm{~s}$ & & $2.01 \mathrm{~s}$ \\
\hline $\mathrm{COCH}_{3}$ & 170.4 & & 170.4 & & & \\
\hline
\end{tabular}

${ }^{a}$ All carbon and proton resonances were assigned on the basis of 2D NMR (DQF-COSY, HSQC, HMBC and ROESY) experiments.

${ }^{\mathrm{b}}$ Signal patterns are unclear due to overlapping.

1 but slightly differ from it in terms of the hydroxycinnamoyl residue. In the ${ }^{1} \mathrm{H}$ NMR spectrum of $\mathbf{3}$, in addition to two characteristic doublets of olefinic protons at $\delta_{\mathrm{H}} 6.32$ and $7.50\left(J_{\mathrm{AX}}=15.9 \mathrm{~Hz}\right)$, four aromatic protons as an $\mathrm{AA}^{\prime} \mathrm{BB}^{\prime}$ system at $\delta_{\mathrm{H}} 7.49(\mathrm{~d}$, $\left.J=8.5 \mathrm{~Hz}, \mathrm{H}-2^{\prime \prime \prime \prime} / 6^{\prime \prime \prime \prime}\right)$ and $6.79(\mathrm{~d}, J=8.5 \mathrm{~Hz}$, $\left.3^{\prime \prime \prime \prime} / 5^{\prime \prime \prime \prime}\right)$ were observed. This data indicated that compound 3 contained trans-coumaroyl instead of trans-caffeoyl as an acyl ester moiety (Klimek, 1988).
These conclusions were also confirmed by 2D NMR experiments (COSY, HSQC and HMBC). Consequently, the structure of compound $\mathbf{2}$ was established as chrysoeriol 7-O-[2'"'-O-caffeoyl- $\beta$-D-glucopyranosyl$(1 \rightarrow 2)$ - $\beta$-D-glucopyranoside] and named ozturkoside $\mathrm{B}$, whereas structure of compound $\mathbf{3}$ was established as chrysoeriol 7-O-[2'"'-O-p-coumaroyl- $6^{\prime \prime \prime}$-O-acetyl$\beta$-D-glucopyranosyl- $(1 \rightarrow 2)$ - $\beta$-D-glucopyranoside] and named ozturkoside $\mathrm{C}$. 
Compounds 4-11 were identified as verbascoside $\mathbf{4}$ (Sticher and Lahloub, 1982; Calis et al., 1984), leucoseptoside A 5 (Miyase et al., 1982) martynoside 6 (Miyase et al., 1982; Calis et al., 1984), 7-epicandicandiol 7 (Gonzales et al., 1981), linearol 8 , sidol 9 (Gonzales et al., 1981; Baser et al., 1996), sideroxol 10 (Baser et al., 1996; Topcu et al., 2002) epoxyisolinearol 11 (Venturella et al., 1975), respectively. The NMR and MS data of the known compounds were consistent with those previously reported.

\section{Experimental}

\subsection{General}

VLC: Silicagel 60, 63-200 $\mu \mathrm{m}$ (Merck). CC: Silicagel 60, 63-200 $\mu \mathrm{m}$ (Merck), Polyamide (Polyvinyl-polypyrrolidone) (Woelm), MPLC: Büchi B-681 glass column packed with LiChroprep RP-18 (Merck) using Lewa M5 peristaltic pump. TLC: Silicagel $60 \mathrm{~F}_{254}$ precoated aluminium plates $(0.2 \mathrm{~mm}$, Merck), Silicagel 60 $\mathrm{F}_{254}$ precoated glass plates $(0.25 \mathrm{~mm}$, Merck). Detection: UV flourescence and spraying $1 \%$ vanillin $/ \mathrm{H}_{2} \mathrm{SO}_{4}$, followed by heating at $105{ }^{\circ} \mathrm{C}$ for $1-2 \mathrm{~min}$. IR spectra: FT-IR spectrometer Perkin-Elmer $1720 \mathrm{X}$. UV spectra: Shimadzu UV-160 A. Negative mode ESI-MS: Varian MAT 731 (EI, $70 \mathrm{eV}$ ). FT-ESI-MS: Bruker APEX III MS. ${ }^{1} \mathrm{H}$ NMR, ${ }^{13} \mathrm{C}$ NMR, DEPT- $135,{ }^{1} \mathrm{H}-{ }^{1} \mathrm{H}$ COSY, ${ }^{13} \mathrm{C}-{ }^{1} \mathrm{H}$ HSQC, ${ }^{13} \mathrm{C}-{ }^{1} \mathrm{H}$ HMBC and ${ }^{1} \mathrm{H}-{ }^{1} \mathrm{H}$ ROESY spectra: Bruker AMX 300, DRX 500, DRX 600. Chemical shifts $\delta$ were given in ppm and coupling constants $J$ in $\mathrm{Hz}$. The spectra were measured in DMSO- $\mathrm{d}_{6}$ for new compounds, $\mathrm{CD}_{3} \mathrm{OD}$ for phenylethanoids and $\mathrm{CDCl}_{3}$ for diterpenoids. The spectra were referenced against residual non-deuterated solvent.

\subsection{Plant material}

Sideritis ozturkii Aytac \& Aksoy was collected from Konya, Derebucak in Southern Anatolia, Turkey, in July 2000. The voucher specimens (HUEF 00281) have been deposited at the Herbarium of the Department of Pharmacognosy, Faculty of Pharmacy, Hacettepe University, Ankara, Turkey.

\subsection{Extraction and isolation}

The air-dried and powdered aerial parts of S. ozturkii $(500 \mathrm{~g})$ were extracted with acetone $(2500 \mathrm{ml} \times 2)$ at room temperature. The combined acetone extracts were dried in vacuo at $40{ }^{\circ} \mathrm{C}$. The total extract $(30 \mathrm{~g})$ was initially fractionated by vacuum liquid chromatography (VLC) on silicagel (petroleum $\rightarrow \rightarrow \mathrm{MeOH}$ ) to give eight main fractions (A-H). Fraction $\mathrm{G}$ was chromatographed on polyamide $\mathrm{CC}\left(\mathrm{H}_{2} \mathrm{O} \rightarrow \mathrm{MeOH}\right)$. Subfraction
G8 which is rich in flavonoids were subjected to MPLC (MeOH: $\mathrm{H}_{2} \mathrm{O} 20: 80 \rightarrow 50: 50$ ) affording compound 1 (25 $\mathrm{mg}$ ), compound 2 (22 mg), compound 3 (55 mg). Subfraction $\mathrm{G} 2$ was separated by MPLC (MeOH:acetonitrile: $\mathrm{H}_{2} \mathrm{O}$ 35:5:60) and then by silicagel CC $\left(\mathrm{CHCl}_{3}: \mathrm{MeOH}: \mathrm{H}_{2} \mathrm{O}\right.$ 90:10:1 $\left.\rightarrow 80: 20: 2\right)$ to give compound $5(8 \mathrm{mg})$, and compound $6(6 \mathrm{mg})$. Subfraction G4 was also identified as compound $4(382 \mathrm{mg})$. Compound $7(8 \mathrm{mg})$, compound $8(32 \mathrm{mg})$, compound 9 (20 $\mathrm{mg})$, compound 10 (28 mg), and compound $11(23 \mathrm{mg})$ were purified by Silica gel CC (cyclohexane: acetone:ethylmethylketone $\quad 90: 10: 0 \rightarrow 80: 15: 5) \quad$ from fraction D.

\subsection{Ozturkoside A (1)}

Amorphous yellow powder $(\mathrm{MeOH})$; $\mathrm{UV} \lambda_{\max } \mathrm{nm}$ (MeOH): 250(sh), 270, 337; ( $\mathrm{NaOMe}): 265,396 ;\left(\mathrm{AlCl}_{3}\right)$ : 264, 297 (sh), 367 (sh), 390; ( $\left.\mathrm{AlCl}_{3}+\mathrm{HCl}\right):$ 278, 297 (sh), 352 (sh), $386 \quad$ (NaOAc): 271, 346, 412 (sh); $\left(\mathrm{NaOAc}+\mathrm{H}_{3} \mathrm{BO}_{3}\right): 268,345 ; \mathrm{IR} v_{\max }(\mathrm{KBr}) \mathrm{cm}^{-1}: 3353$ $(\mathrm{OH}), 1715$ (est. $\mathrm{C}=\mathrm{O}), 1657(\gamma$-pyrone $\mathrm{C}=\mathrm{O}), 1602$, 1516 (arom. ring); ${ }^{1} \mathrm{H}$ NMR (600 MHz; DMSO-d 6 ) (see Table 1); ${ }^{13} \mathrm{C}$ NMR (150 MHz; DMSO-d 6 ) (see Table 1); ESI-MS $(m / z)$ negative ion mode: $827[\mathrm{M}-\mathrm{H}]^{-}$, $\mathrm{C}_{39} \mathrm{H}_{40} \mathrm{O}_{20}$. FT-ESI-MS $(\mathrm{m} / \mathrm{z}): 851.20368[\mathrm{M}+\mathrm{Na}]^{+}$, $\mathrm{C}_{39} \mathrm{H}_{40} \mathrm{O}_{20} \mathrm{Na}$ (calc. 851.20105).

\subsection{Ozturkoside B (2)}

Amorphous yellow powder $(\mathrm{MeOH})$; UV $\lambda_{\max } \mathrm{nm}$ $(\mathrm{MeOH}): 250$ (sh), 270, 336; (NaOMe): 260, 392; $\left(\mathrm{AlCl}_{3}\right): 263,298$ (sh),368 (sh), 389; $\left(\mathrm{AlCl}_{3}+\mathrm{HCl}\right): 278$, 297 (sh), 353 (sh), 385; (NaOAc): 270, 345, 412 (sh); $\left(\mathrm{NaOAc}+\mathrm{H}_{3} \mathrm{BO}_{3}\right): 269,345 ; \mathrm{IR} v_{\max }(\mathrm{KBr}) \mathrm{cm}^{-1}: 3399$ $(\mathrm{OH}), 1714$ (est. $\mathrm{C}=\mathrm{O}$ ), 1659 ( $\gamma$-pyrone $\mathrm{C}=\mathrm{O}), 1597$, 1499 (arom. ring); ${ }^{1} \mathrm{H}$ NMR (500 MHz; DMSO-d 6 ) (see Table 1); ${ }^{13} \mathrm{C}$ NMR (125 MHz; DMSO-d 6 ) (see Table 1); ESI-MS $(\mathrm{m} / z)$ negative ion mode: $785[\mathrm{M}-\mathrm{H}]^{-}$, $\mathrm{C}_{37} \mathrm{H}_{38} \mathrm{O}_{19}$. FT-ESI-MS $(\mathrm{m} / \mathrm{z}): 809.19169[\mathrm{M}+\mathrm{Na}]^{+}$, $\mathrm{C}_{37} \mathrm{H}_{38} \mathrm{O}_{19} \mathrm{Na}$ (calc. 809.19048).

\subsection{Ozturkoside C (3)}

Amorphous yellow powder $(\mathrm{MeOH})$; UV $\lambda_{\max } \mathrm{nm}$ $(\mathrm{MeOH}): 250$ (sh), 271, 336; (NaOMe): 265, 392; $\left(\mathrm{AlCl}_{3}\right): 264,297$ (sh), 368 (sh), 389; $\left(\mathrm{AlCl}_{3}+\mathrm{HCl}\right): 278$, 296 (sh), 353 (sh), 386; (NaOAc): 271, 347, 412 (sh); $\left(\mathrm{NaOAc}+\mathrm{H}_{3} \mathrm{BO}_{3}\right): 270,346$; IR $v_{\max }(\mathrm{KBr}) \mathrm{cm}^{-1}: 3396$ $(\mathrm{OH}), 1714$ (est. $\mathrm{C}=\mathrm{O}), 1661(\gamma$-pyrone $\mathrm{C}=\mathrm{O}), 1604$, 1515 (arom. ring); ${ }^{1} \mathrm{H}$ NMR (300 MHz; DMSO-d $\mathrm{d}_{6}$ ) (see Table 1); ${ }^{13} \mathrm{C}$ NMR (75 MHz; DMSO-d 6 ) (see Table 1); ESI-MS $(m / z)$ negative ion mode: $811[\mathrm{M}-\mathrm{H}]^{-}$, $\mathrm{C}_{39} \mathrm{H}_{40} \mathrm{O}_{19}$. FT-ESI-MS $(\mathrm{m} / \mathrm{z}): 835.21183[\mathrm{M}+\mathrm{Na}]^{+}$, $\mathrm{C}_{39} \mathrm{H}_{40} \mathrm{O}_{19} \mathrm{Na}$ (calc. 835.20613). 


\subsection{Verbascoside (4)}

Amorphous yellow powder $(\mathrm{MeOH}) ; \mathrm{UV} \lambda_{\max } \mathrm{nm}$ $(\mathrm{MeOH}): 220,234(\mathrm{sh}), 290,329$; IR $v_{\max }(\mathrm{KBr}) \mathrm{cm}^{-1}$ : 3392, 1699, 1631, 1604, 1523.

\subsection{Leucoseptoside A (5)}

Amorphous yellow powder $(\mathrm{MeOH})$; $\mathrm{UV} \lambda_{\max } \mathrm{nm}$ (MeOH): 219, 234 (sh), 289, 327; IR $v_{\max }(\mathrm{KBr}) \mathrm{cm}^{-1}$ : 3380, 1700, 1632, 1599, 1516.

\subsection{Martynoside (6)}

Amorphous yellow powder $(\mathrm{MeOH})$; $\mathrm{UV} \lambda_{\max } \mathrm{nm}$ (MeOH): 219, 234 (sh), 288, 327; IR $v_{\max }(\mathrm{KBr}) \mathrm{cm}^{-1}$ : 3399, 1700, 1634, 1595, 1516.

\subsection{7-Epicandicandiol (7)}

Colorless needles $\left(\mathrm{CHCl}_{3}\right)$; UV $\lambda_{\max } \mathrm{nm}\left(\mathrm{CHCl}_{3}\right)$ : 248; IR $v_{\max }(\mathrm{KBr}) \mathrm{cm}^{-1}: 3390,2929,1646,871$; EI-MS $(m / z): 304.3[\mathrm{M}]^{+}, 286.3\left[\mathrm{M}-\mathrm{H}_{2} \mathrm{O}\right]^{+}, 271.2[\mathrm{M}-$ $\left.\mathrm{H}_{2} \mathrm{O}-\mathrm{CH}_{3}\right]^{+}, \mathrm{C}_{20} \mathrm{H}_{32} \mathrm{O}_{2}$.

\subsection{Linearol (8)}

Colorless prisms $\left(\mathrm{CHCl}_{3}\right)$; UV $\lambda_{\max } \mathrm{nm}\left(\mathrm{CHCl}_{3}\right)$ : 247,5; IR $v_{\max }(\mathrm{KBr}) \mathrm{cm}^{-1}: 3462,2930,1655,875,1715$; EI-MS $(m / z): 362.3[\mathrm{M}]^{+}, 344.3\left[\mathrm{M}-\mathrm{H}_{2} \mathrm{O}\right]^{+}, 326.3[\mathrm{M}-$ $\left.2 \mathrm{H}_{2} \mathrm{O}\right]^{+}, \mathrm{C}_{22} \mathrm{H}_{34} \mathrm{O}_{4}$

\subsection{Sidol (9)}

White resin $\left(\mathrm{CHCl}_{3}\right) ; \mathrm{UV} \lambda_{\max } \mathrm{nm}\left(\mathrm{CHCl}_{3}\right): 247,5 ; \mathrm{IR}$ $v_{\max }(\mathrm{KBr}) \mathrm{cm}^{-1}: 3462,2930,1655,875,1715$; ESI-MS $(\mathrm{m} / \mathrm{z})$ positive ion mode: $385.2[\mathrm{M}+\mathrm{Na}]^{+}, \mathrm{C}_{22} \mathrm{H}_{34} \mathrm{O}_{4}$

\subsection{Sideroxol (10)}

White resin $\left(\mathrm{CHCl}_{3}\right) ; \mathrm{UV} \lambda_{\max } \mathrm{nm}\left(\mathrm{CHCl}_{3}\right): 248$; IR $v_{\max }(\mathrm{KBr}) \mathrm{cm}^{-1}: 3432,2927,1043$; ESI-MS $(m / z)$ positive ion mode: $343.2[\mathrm{M}+\mathrm{Na}]^{+}, \mathrm{C}_{20} \mathrm{H}_{32} \mathrm{O}_{3}$.

\subsection{Epoxyisolinearol (11)}

White resin $\left(\mathrm{CHCl}_{3}\right)$; UV $\lambda_{\max } \mathrm{nm}\left(\mathrm{CHCl}_{3}\right): 247,5 ; \mathrm{IR}$ $v_{\max }(\mathrm{KBr}) \mathrm{cm}^{-1}: 3432,2927,1043,1730$; EI-MS $(\mathrm{m} / z)$ : $378.3[\mathrm{M}]^{+}, 396.3\left[\mathrm{M}+\mathrm{H}_{2} \mathrm{O}\right]^{+}, \mathrm{C}_{22} \mathrm{H}_{34} \mathrm{O}_{5}$.

\section{Acknowledgements}

The authors are grateful to Dr. Akito Nagatsu (Nagoya City University, Graduate School of Pharmaceutical Sciences, Mizuho-ku, Nagoya, Japan) for FTESI-MS, and Dr. Hayri Duman (Gazi University, Faculty of Science, Department of Botany, Ankara, Turkey) for the authentification of the plant specimen.

\section{References}

Akcos, Y., Ezer, N., Calis, I., Demirdamar, R., Tel, B.C., 1999. Polyphenolic compounds of Sideritis lycia and their anti-inflamamatory activity. Pharm. Biol. 37, 118-122.

Aytac, Z., Aksoy, A., 2000. A new Sideritis species (Labiatae) from Turkey. Flora Mediterranea 10, 181-184.

Baser, K.H.C., Bondi, M.L., Bruno, M., Kırımer, N., Piozzi, F., Tümen, G., Vassallo, N., 1996. An ent-kaurane from Sideritis huber morathii. Phytochemistry 43, 1293-1295.

Baser, K.H.C., 2002. Aromatic biodiversity among the flowering plant taxa of Turkey. Pure Appl. Chem. 74, 527-545.

Baytop, T., 1999. Therapy with Medicinal Plants in Turkey (Past and Present). Nobel Tip Publications, Istanbul.

Calis, I., Lahloub, M.F., Rogenmoser, E., Sticher, O., 1984. Isomartynoside, a phenylpropanoid glycoside from Galeopsis pubescens. Phytochemistry 23, 2313-2315.

Calis, I., Kirmizibekmez, H., Ersoz, T., Saracoglu, I., Donmez, A.A., Mitova, M., Handjieva, N., Popov, S., 2002. Iridoid, phenylethanoid and flavonoid glycosides from Phlomis sintenisii. Acta Pharm. Turc. 44, 195-203.

Ezer, N., Sakar, M.K., Rodriguez, B., De la Torre, M.C., 1992. Flavonoid glycosides and a phenylpropanoid glycoside from Sideritis perfoliata. Int. J. Pharmacogn. 30, 61-65.

Ezer, N., Akcos, Y., 1995. Flavonoids from Sideritis lycia. H.U. J. Fac. Pharm. 15, 81-87.

Ezer, N., Vila, R., Canigueral, S., Adzet, T., 1996. Essential oil composition of four Turkish species of Sideritis. Phytochemistry 41, 203-205.

Gonzales, A.G., Fraga, M.B., Hernandez, M.G., Hanson, J.R., 1981. The ${ }^{13} \mathrm{C}$ NMR spectra of some ent-18-hydroxykaur-16-enes. Phytochemistry 20, 846-847.

Klimek, B., 1988. Acylated 6-hydroxyluteolin diglucosides from Globularia elongata. Phytochemistry 27, 255-258.

Mabry, T.J., Markham, K.R., Thomas, M.B., 1970. The Systematic Identificaion of Flavonoids. Springer-Verlag, Berlin.

Miyase, T., Koizumi, A., Ueno, A., Noro, T., Kuroyanagi, M., Fukushima, S., Akiyama, Y., Takemoto, T., 1982. Studies on the acyl glycosides from Leucoseptrum japonicum (Miq.) Kitamura et Murata. Chem. Pharm. Bull. 30, 2732-2737.

Obon de Castro, C., Rivera-Nunez, D., 1994. A Taxonomic Revision of the Section Sideritis (Genus Sideritis) (Labiatae). J. Cramer, Berlin-Stuttgart.

Sticher, O., Lahloub, M.F., 1982. Phenolic glycosides of Paulownia tomentosa bark. Planta Med. 46, 145-148.

Topcu, G., Gören, A.C., Kilic, T., Yildiz, Y.K., Tümen, G., 2002. Diterpenes from Sideritis trojana. Nat. Prod. Lett. 16, 33-37.

Venturella, P., Bellino, A., Piozzi, F., 1975. Diterpenes from Sideritis theezans. Phytochemistry 14, 1451-1452. 